\title{
Anatomia do lenho de Uncaria guianensis e U. tomentosa (Rubiaceae) do Estado do Acre, Brasil
}

\author{
Percy A. ZEVALlOS POLLITO1, Mario TOMAZELLO²
}

\section{RESUMO}

As plantas de Uncaria guianensis e U. tomentosa são lianas trepadeiras, arbustos trepadores ou rasteiros ascendentes que ocorrem em amplas áreas da Amazônia brasileira e países da América Central e do Sul. Possuem excepcional importância medicinal, com sua casca, lenho e folhas utilizadas pelos povos amazônicos e pela indústria farmacêutica. Face a necessidade da identificação das plantas em condiçôes de campo e de laboratório, o presente trabalho descreveu a estrutura anatômica do lenho de Uncaria guianensis e $U$. tomentosa, ocorrentes no Estado do Acre, Brasil. Os resultados mostraram que o lenho das espécies é característico da família Rubiaceae, a exceção dos vasos arredondados, grandes e solitários, parenquima axial difuso e largura dos raios que são próprios das lianas.

\section{PALAVRAS-CHAVE}

Anatomia do lenho, liana, Rubiaceae, Uncaria guianensis, Uncaria tomentosa.

\section{Wood anatomy of Uncaria guianensis $e \mathrm{U}$. tomentosa (Rubiaceae) from the State of Acre, Brazil}

\section{ABSTRACT}

Uncaria guianensis and $\mathrm{U}$. tomentosa are climbing lianas, bush lianas occuring in large areas of Brazilian Amazon and Central and South America countries. The species presented exceptional medicinal importance, with its bark, wood and leaves used by Amazon native people and pharmaceutical industries. Considering the importance of the species identification in field and laboratory conditions, the present work described the anatomical structure of Uncaria guianensis and $\mathrm{U}$. tomentosa, which occurs in State of Acre, Brazil. The results showed that the wood of the species was characteristic of the Rubiaceae family, except the rounded vessel, greater and solitary, parenchyma axial diffuse and width of the rays, proper of lianas.

\section{KEY WORDS}

wood anatomy, liana, Rubiaceae, Uncaria guianensis, Uncaria tomentosa.

${ }^{1}$ Faculdade de Ciências Florestais/Universidade Nacional Agrária La Molina, C.P. 456. Lima/Peru (pazpollito@lamolina.edu.pe);

2Departamento de Ciências Florestais-ESALQ/Universidade de São Paulo, C.P. 09. 13418-900. Piracicaba, SP/Brasil (mtomazel@esalq.usp.br) 


\section{INTRODUÇÃO}

O gênero Uncaria é representado por cerca de 60 espécies de lianas distribuídas principalmente na África e Ásia (Obregón, 1995; Steyermark, 1974) sendo que, Uncaria guianensis e $U$. tomentosa (Ridsdale, 1978; Zavala \& Zevallos, 1996), ocorrem em amplas áreas da Amazônia brasileira e demais países da América Central (Guatemala, Belize, Honduras, El Salvador, Nicarágua, Costa Rica, Panamá) e do Sul (Colômbia, Venezuela, Guiana, Equador, Peru e Bolívia), indicadas como seu centro de origem (Villachica et al., 1998; Zevallos et al., 2000) (Fig. 1). As plantas de Uncaria são, em geral, lianas, arbustos- trepadeiras ou rasteiras ascendentes, com um par de espinhos, originados de pedúnculos abortivos, utilizados para galgar a copa das árvores (Ridsdale, 1978; Reinhard, 1999; Zevallos et al., 2000), denominadas de "unha de gato" por Steyermark (1974). Possuem folhas simples e opostas, lâmina ovada, elíptica, consistência cartácea ou papirácea e um par de estípulas interpeciolares, livres na base, deltóide, obovada, com tomentos na superfície da folha em Uncaria tomentosa e grabra em U. guianensis (Zavala \& Zevallos, 1996). A casca, lenho e folhas dessas plantas têm sido usadas pelos povos amazônicos (ex.: os Ashaninkas do Peru) para o tratamento de inúmeras enfermidades (gastrite, úlcera, diarréia, artrite, câncer, etc.) e como abortivo (Zevallos et al., 2000), corroborando os resultados das pesquisas realizadas pela indústria farmacêutica (Keplinger et al., 1999). Dentre os usos medicinais atuais das plantas de Uncaria mencionam-se suas propriedades como estimulantes do sistema imunológico e contra o câncer, além da aplicação contra a AIDS (Lock de Ugaz, 1994; Urrunaga, 1994; Keplinger et al., 1999) e ação anticonceptiva (TCA, 1995) e afrodisíaca (IPSS, 1997). A seiva da unha de gato, pelas suas propriedades energéticas e nutritivas, é utilizada pelos povos das florestas (Cabieses, 1997). A indústria de medicamentos naturais

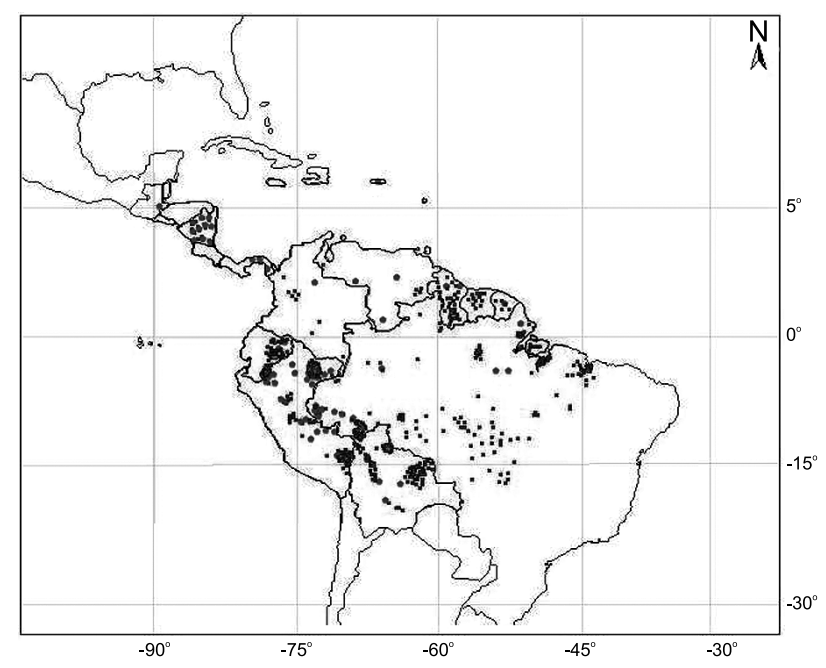

Figura 1 - Distribução geográfica das espécies do gênero Uncaria na América Latina. fabrica remédios para doenças cardiovasculares (Wang, 1989; Huang, 1999) renais, ginecológicas, gastrointestinais, hepáticas, reumatológicas e reprodutivas (Keplinger, 1999), imune estimulante, antimicrobianos e antivirais (Aquino et al., 1989) além de aumentar a resistência dos tecidos (Desmarchelier $e t$ al., 1997). Apesar da extensa bibliografia médica, as informações sobre a anatomia das duas espécies de Uncaria concentram-se na sua casca (Silva et al., 1998; Lindorf, 2001). Em relação a madeira existem apenas descrições gerais do lenho na Amazônia peruana (Chavesta \& Gonzáles, 1996; Zevallos et al., 2002).

Pelo exposto, o presente trabalho visa caracterizar a estrutura anatômica do lenho das plantas de Uncaria guianensis e $U$. tomentosa como subsídio para a identificação das espécies em campo e em laboratório, para estudos taxonômicos, medicinais e ecológicos, dentre outros.

\section{MATERIAL E MÉTODOS}

Localização das coletas: o material botânico de Uncaria guianensis (Aubl.) J.F. Gmel e U. tomentosa (Willd. Ex Roem. \& Schult.) DC. foi coletado nos trabalhos de campo realizados em 18 municípios do Estado do Acre, a saber: Acrelândia, Assis Brasil, Brasiléia, Bujari, Capixaba, Cruzeiro do Sul, Epitaceolândia, Feijó, Mâncio Lima, Manoel Urbano, Plácido de Castro, Porto Walter, Rio Branco, Rodrigues Alves, Senador Guiomard, Sena Madureira, Tarauacá e Xapuri (Fig. 2).

Amostragem e preparo do material: Para Uncaria guianensis foram coletadas amostras do lenho de oito plantas com 10 repetições cada e para Uncaria tomentosa de quatro plantas com 10 repetiçōes. As amostras foram cortadas na região basal do caule das plantas, incluindo a coleta de material botânico. Em laboratório as amostras do lenho foram preparadas para os estudos macro e microscópicos, inicialmente, pelo cortes histológicos dos corpos de prova $(1.5 \times 1.5 \times 1.5 \mathrm{~cm})$ orientados nos planos transversal, longitudinal radial e tangencial (Johansen, 1940; Sass, 1951).

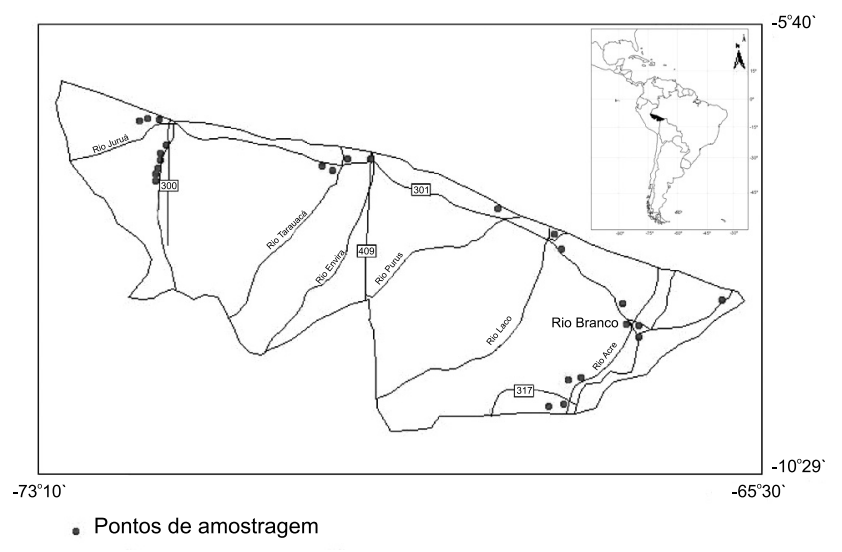

Figura 2 - Localização dos pontos de amostragem no Estado do Acre 
Tabela 1 - Diferênças na estrutura anatômica do lenho das plantas de Uncaria guianensis e $U$. tomentosa

\begin{tabular}{|c|c|c|}
\hline CARACTERISTICAS & U. guianensis & U. tomentosa \\
\hline \multirow[t]{4}{*}{ Cor } & Vermelho-amarelado & Avermelhado \\
\hline & Bege-avermelhado & Vermelho-amarelado \\
\hline & Rosa-amarelado & Laranja-amarelado \\
\hline & & Rosa-avermelhado \\
\hline Densidade básica & Média/baixa & Baixa \\
\hline Resistência ao corte & Moderadamente dura & Macia \\
\hline \multicolumn{3}{|l|}{ Vasos } \\
\hline \multicolumn{3}{|l|}{ Agrupamento dos vasos (\%) } \\
\hline Solitários & 64 & 96 \\
\hline Múltiplos & $36(2-3)$ & $4[2-(3)]$ \\
\hline Frequiência (n\% mm²) & $3-13$ & $3-8$ \\
\hline \multicolumn{3}{|l|}{ Diâmetro tangencial $(\mu \mathrm{m})$} \\
\hline Mínimo & 107 & 120 \\
\hline Médio & 309 & 334 \\
\hline Máximo & 453 & 613 \\
\hline Desvio padrão & 91.48 & 110.62 \\
\hline \multicolumn{3}{|l|}{ Comprimento $(\mu \mathrm{m})$} \\
\hline Mínimo & 293 & 240 \\
\hline Médio & 599 & 509 \\
\hline Máximo & 826 & 867 \\
\hline Desvio padrão & 125.61 & 107.48 \\
\hline \multicolumn{3}{|c|}{ Diâmetro das pontoações intervasculares } \\
\hline Mínimo & 4 & 5 \\
\hline Médio & 6 & 6 \\
\hline Máximo & 7 & 8 \\
\hline Desvio padrão & 0.36 & 0.91 \\
\hline \multicolumn{3}{|c|}{ Diâmetro das pontoações radio-vasculares } \\
\hline Mínimo & 4 & 4 \\
\hline Médio & 6 & 6 \\
\hline Máximo & 7 & 7 \\
\hline Desvio padrão & 0.75 & 0.98 \\
\hline \multicolumn{3}{|c|}{ Diâmetro das pontoações parênquimo-vasculares } \\
\hline Mínimo & 4 & 5 \\
\hline Médio & 6 & 6 \\
\hline Máximo & 7 & 8 \\
\hline Desvio padrão & 0.36 & 0.91 \\
\hline \multicolumn{3}{|c|}{ Diâmetro das pontuações das fibras } \\
\hline Mínimo & 4 & 4 \\
\hline Médio & 6 & 6 \\
\hline Máximo & 8 & 8 \\
\hline Desvio padrão & 0.77 & 0.58 \\
\hline \multicolumn{3}{|l|}{ Fibras } \\
\hline \multicolumn{3}{|l|}{ Comprimento ( $\mu \mathrm{m})$} \\
\hline Mínimo & 1110 & 509 \\
\hline Médio & 1483 & 980 \\
\hline Máximo & 1910 & 1760 \\
\hline Desvio padrão & 101 & 110 \\
\hline Largura $(\mu \mathrm{m})$ & $19-44$ & $19-47$ \\
\hline Espessura da parede $(\mu \mathrm{m})$ & $9-28$ & $16-25$ \\
\hline
\end{tabular}

\begin{tabular}{lll}
\hline \multicolumn{2}{l}{ Tabela 1 - continuação } \\
\hline $\begin{array}{l}\text { Parênquima radial (raios) } \\
\text { Largura (\%) }\end{array}$ \\
Unisseriado & 66 & 63 \\
Multiserriado & $34(2-3)$ & $37(2-5)$ \\
\hline
\end{tabular}

As exsicatas, coletadas do lenho foram: Uncaria guianensis: Zevallos (2914; 2922; 2925; 2933; 2942; 2959; 2964; 2976) e de U. tomentosa: Zevallos (2931; 2936; 2958; 2973), e encontram-se depositadas nos herbários do Parque Zoobotânico da Universidade Federal do Acre (HPZ) e as duplicatas no herbário da Escola Superior de Agricultura Luiz de Queiroz da Universidade de São Paulo (ESA). A identificação do material botânico foi realizada de acordo com a metodologia de Radford et al. (1974).

Análise da estrutura macroscópica do lenho: para a observação da superfície do lenho, os corpos de prova foram inicialmente cortados com navalha em micrótomo de deslize e, em seguida, polidos pela abrasão em pedra de afiação de granulação fina umedecida em água e em tecido macio para propiciar o contraste dos elementos anatômicos. A densidade básica foi obtida pela massa seca do lenho (em estufa) pelo volume verde (saturado em água até peso constante). A estrutura anatômica macroscópica do lenho das duas espécies de Uncaria, observada sob microscópio estereoscópico, foi descrita atendendo as normas da COPANT (1974) e Coradin \& Muniz (1992).

Análise da estrutura microscópica do lenho: a estrutura microscópica do lenho foi analisada através de lâminas histológicas permanentes (Johansen, 1940; Sass, 1951) e células dissociadas pelo processo de maceração (Franklin, 1937; Berlyn, 1976). Corpos de prova do lenho das plantas das duas espécies de Uncaria foram (i) imersos em água para a saturação completa e, em seguida, em água a ebulição para amolecimento prévio; (ii) fixados em micrótomo de deslize foram obtidas seçôes histológicas finas (espessura 12-14 $\mu \mathrm{m}$ ) dos 3 planos, (iii) as seçôes foram clarificadas (água sanitária), lavadas (água destilada) e desidratadas (álcool 30 a 50\%), coradas (safranina e astra-blue, 30 a 70\%), desidratadas (série alcóolica, 50 a 100\%; acetato e álcool, 1:1; acetato de $\mathrm{N}$-butila), selecionadas e montadas em lâmina de vidro com bálsamo do Canadá. Para o processo de maceração, segmentos do lenho foram transferidos para tubos de ensaio com água destilada e, em seguida, com solução macerante (ácido acético glacial e água oxigenada 120 vol, levados a estufa $\left(60^{\circ} \mathrm{C}, 48 \mathrm{~h}\right)$ que, depois de escoada, foram lavados com água. Para o estudo da morfologia e a avaliação das dimensōes, as células dissociadas do lenho foram coradas com safranina e montadas em lâminas semi-permanentes com glicerina, sob microscópio acoplado a câmera de vídeo e sistema de análise de imagens. A descrição da estrutura anatômica microscópica do lenho das duas espécies de Uncaria foi conduzida de acordo com as normas da COPANT (1974) e da IAWA (1989). 


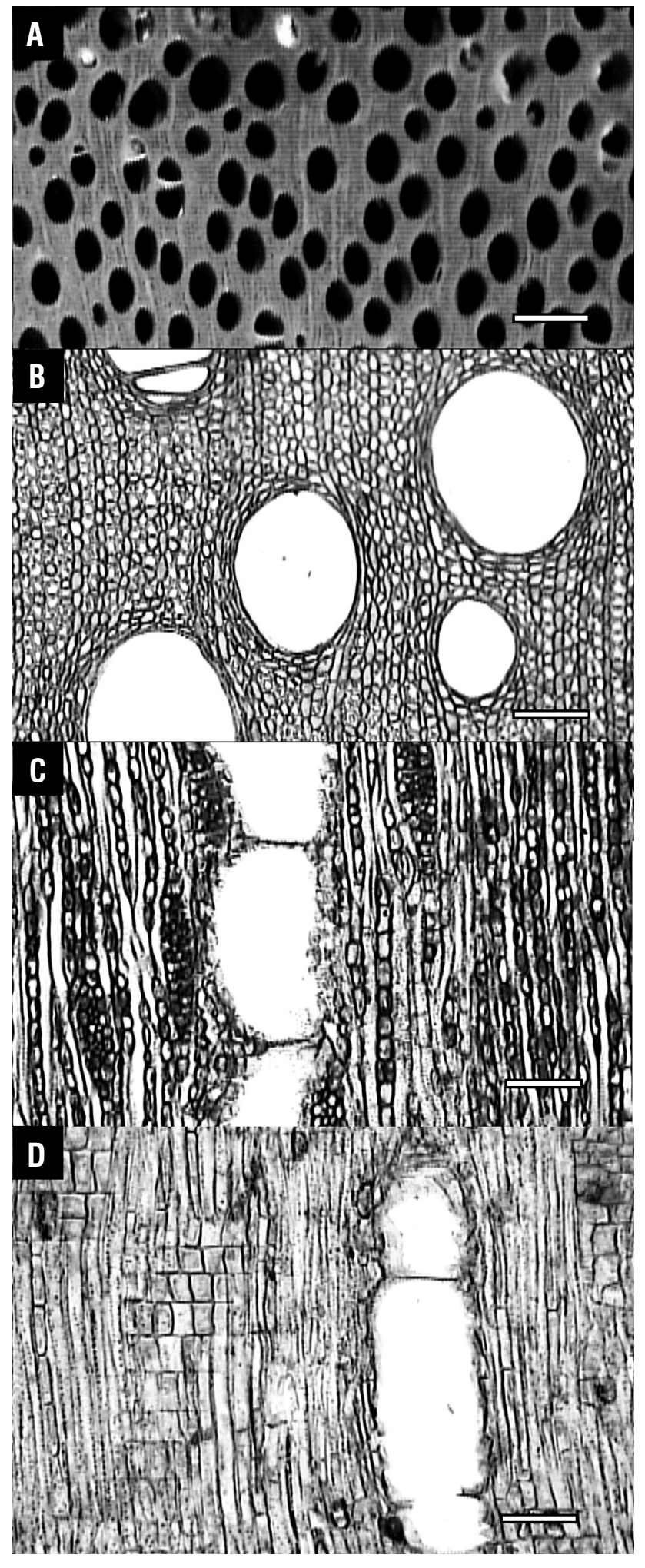

Figura 3 - Uncaria guianensis - (a) Seção transversal (10 X), (b) transversal, (c) longitudinal tangencial, (d) Iongitudinal radial (50X). Escala: a: $1 \mathrm{~mm} ; \mathrm{a}, \mathrm{b}, \mathrm{c}, \mathrm{d}: 250 \mu \mathrm{m}$.

\section{RESULTADOS E DISCUSSÃO}

As observaçôes sobre as características organolépticas do lenho, os nomes vulgares aplicados em sua extensa área de ocorrência e a descrição da estrutura anatômica do lenho das duas espécies de Uncaria são apresentados.

\section{Uncaria guianensis}

Nomes vulgares: Brasil: Acre: anzol-de-lontra, cipó, cipóanzol-de-lontra, espara-aí, esperái, unha-de-gato; Amapá: jupindá, mão-de-gato; Amazonas: espera-aí, unha-de-gato; Maranhão: maracuçumé, paruá-cipó; Mato Grosso: jupindá, unha-de-onça; Pará: cuerussu, jupídá-do-vermelho, pau-d’arco, unha-de-gato; Rondônia: unha-de-lontra. Bolívia: cacatao, misijuni, unha-de-gato. Peru: ancajsillo, ancauacu, auri huasca, garabata, garabata casha, garabato, garabato colorado, garabatocasha, huarinhuasca, jijyúwamyuúho, kug kuukjagki, tambo huasca, toroñ, uña de gato, uña de gavilán, unganangui, yamayakat. Suriname: sipaliwini. Venezuela: uña de gavilán.

Caracterização anatômica do lenho: Cerne indistinto do alburno, de cor vermelho-amarelado, bege-avermelhado ou rosaavermelhado, brilho moderado, odor imperceptível, densidade básica média/baixa, resistência ao corte moderadamente dura, grã direita, textura média/grossa. Camadas de crescimento indistintas ou pouco distintas. Vasos visíveis a olho nu; difusos; solitários (64\%) e múltiplos (36\%) de 2-3 células, freqüência 3$13 / \mathrm{mm}^{2}$; diâmetro tangencial $107-453 \mu \mathrm{m}$, média $309 \mu \mathrm{m} \mathrm{e}$ forma arredondada; comprimento 293-826 $\mu \mathrm{m}$, média $599 \mu \mathrm{m}$; apêndices curtos, menor a $1 / 3$ do comprimento do vaso e em ambas extremidades; placas de perfuração simples; depósitos de cristais distintos; pontoações intervasculares areoladas, alternas, arredondadas, abundantes, ornamentação ausente, diâmetro tangencial 4-7 $\mu \mathrm{m}$, média $6 \mu \mathrm{m}$; pontoaçōes raio-vasculares, areoladas, alternas, arredondadas, abundantes, diâmetro tangencial 4-7 $\mu \mathrm{m}$, média de $6 \mu \mathrm{m}$; pontoaçōes parênquimovasculares semelhantes às intervasculares. Fibras não septadas, com pontoaçôes distintamente areoladas maior de $3 \mu \mathrm{m}$; curtas a longas, comprimento 1110-1910 $\mu \mathrm{m}$, média $1483 \mu \mathrm{m}$; largura média 19-44 $\mu \mathrm{m}$; espessura da parede delgada com lume de 9$28 \mu \mathrm{m}$; pontoaçōes alternas, arredondadas, abundantes nas seçōes radial e tangencial, com diâmetro tangencial 4-8 $\mu \mathrm{m}$, média 6 $\mu \mathrm{m}$. Parênquima axial invisível a olho nu e indistinto sob lente (10x); apotraqueal difuso em agregados formando linhas; células do tipo seriado. Parênquima radial (raios) invisível sob lente (10x), unisseriados $(66 \%)$ e multisseriados (34\%), com mais de 2-3 células em largura; altura 3-12 mm, média $7.52 \mathrm{~mm}$; freqüência 6-20/mm, média 13,5/mm; heterocelulares, com 8-9 fileiras (32\%) de células eretas no extremo superior, no centro 10-13 fileiras (44\%) de células procumbentes e 5-8 fileiras (24\%) de células eretas no extremo inferior; com 7-30 linhas de células; largura variando de 27-107 $\mu \mathrm{m}$ (Fig. 3, Tab. 1). 

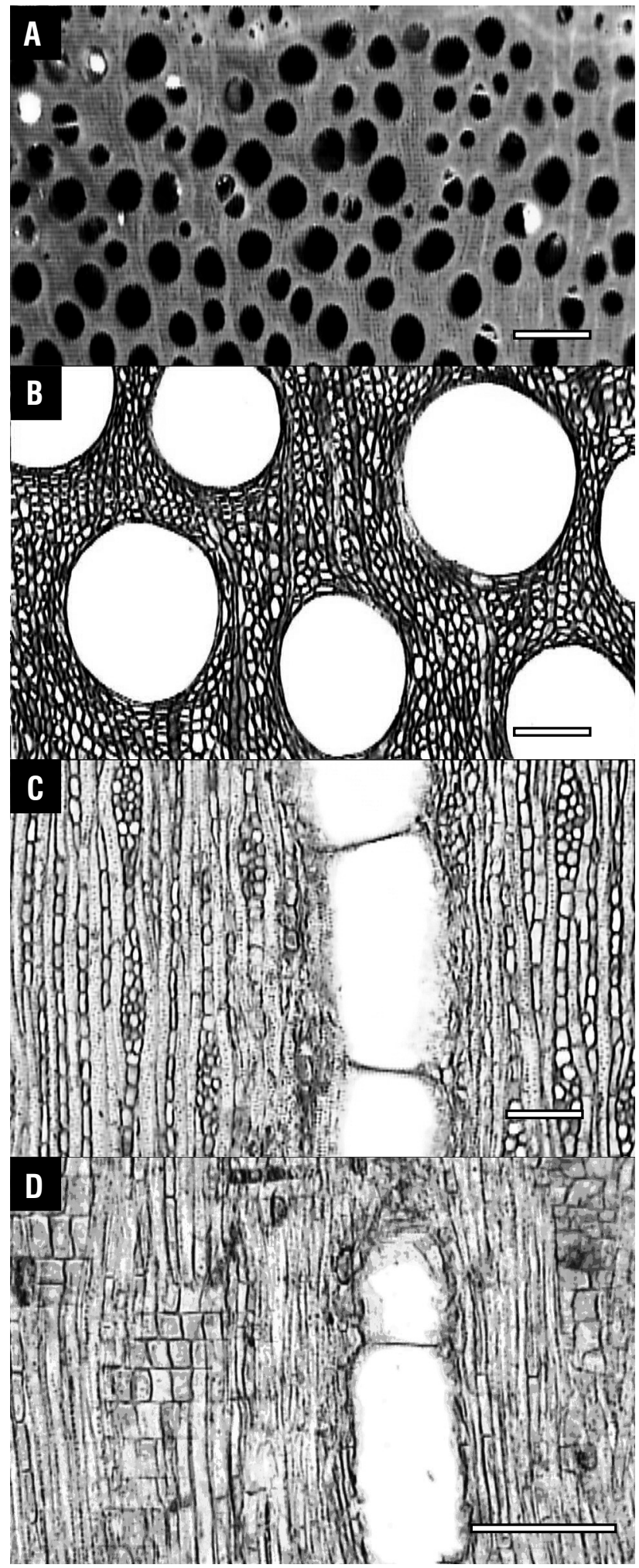

Figura 4 - Uncaria tomentosa - (a) Seção transversal (10X), (b) transversal, (c) longitudinal tangencial, (d) longitudinal radial (50X). Escala: a: $1 \mathrm{~mm} ; \mathrm{b}, \mathrm{c}, \mathrm{d}: 250 \mu \mathrm{m}$.

\section{Uncaria tomentosa}

Nomes vulgares: Brasil: Acre: espera-aí, unha-de-gato; Amapá: jupinda, jupindá; Amazonas: espera-aí, unha-de-gato. Bolívia: bereoquida. Peru: garabato amarillo, jipotatsa, misho mentis, paotati mösha, samento, toroñ, tsachk, uña de gato, unganangui.

Caracterização anatômica do lenho: Cerne indistinto do alburno, de cor avermelhado, vermelho-amarelado, laranjaamarelado, a rosa-avermelhado, brilho moderado, odor imperceptível, densidade básica baixa, resistência ao corte macia, grã direita, textura média/grossa. Camadas de crescimento indistintas ou pouco distintas. Vasos visíveis a olho nu; difusos; solitários (96\%) e múltiplos (4\%) de 2-3 células, freqüência 38/ $\mathrm{mm}^{2}$; diâmetro tangencial 120-163 $\mu \mathrm{m}$, média $334 \mu \mathrm{m}$, forma arredondada; comprimento 240-867 $\mu \mathrm{m}$, média $509 \mu \mathrm{m}$; apêndice curto, menor a $1 / 3$ do comprimento do vaso e em ambas extremidades; placas de perfuração simples; depósitos de cristais distintos; pontoaçóes intervasculares areoladas, alternas, arredondadas, abundantes, ornamentação ausente, diâmetro tangencial 5-8 $\mu \mathrm{m}$, média $6 \mu \mathrm{m}$; pontoaçōes raio-vasculares aeroladas, alternas, arredondadas, abundantes, diâmetro tangencial 4-7 $\mu \mathrm{m}$, média de $6 \mu \mathrm{m}$; pontoações parênquimovasculares semelhantes às intervasculares. Fibras não septadas, com pontoaçôes distintamente areoladas maior de $3 \mu \mathrm{m}$; curtas a longas, comprimento 509-1760 $\mu \mathrm{m}$, média $980 \mu \mathrm{m}$; largura média 19-47 $\mu \mathrm{m}$; espessura da parede delgada a espessa, com lume de 16-25 $\mu \mathrm{m}$; pontoaçôes alternas, arredondadas, muito abundantes nas seçôes radial e tangencial, com diâmetro tangencial 4-8 $\mu \mathrm{m}$, média $6 \mu \mathrm{m}$. Parênquima axial invisível a olho nu e indistinto sob lente (10x); apotraqueal difuso em agregados formando linhas; células do tipo seriado. Parênquima radial (raios) invisível sob lente $(10 \mathrm{x})$, unisseriado $(63 \%)$ e multisseriado (37\%), com mais de 2-5 células em largura; altura 3-12 mm, média $6,69 \mathrm{~mm}$; freqüência $3-16 / \mathrm{mm}$, média $9 / \mathrm{mm}$; heterocelulares, com 4-6 fileiras (20\%) de células eretas na extremidade superior, no centro 10-11 fileiras (45\%) de células procumbentes e $8-9$ fileiras $(35 \%)$ de células eretas na extremidade inferior; com 7-42 linhas de células; largura variando de 20-107 $\mu \mathrm{m}$ (Fig. 4, Tab.1).

As duas espécies de Uncaria apresentaram lenho avermelhado, com variaçóes do amarelado ao rosado ou bege ao alaranjado, característica da família Rubiaceae (Détienne \& Jacquet, 1983) e distintas das de Pentagonia e Chomelia, de cor clara, do branco ao bege claro (Freitas et al., 1992). O odor indistinto, grã direita, textura médio-grossa, brilho moderado e densidade básica baixa a média, resistência ao corte de mole a moderadamente dura (U. guianensis) corroboram a descrição apresentada por Zevallos et al. (2002) e Chavesta \& Gonzáles (1996). As características anatômicas do lenho das duas espécies de Uncaria são coincidentes com as descritas para outras espécies da família (Jansen et al., 2002; Record \& Hess, 1949; Freitas et 
al., 1992; Detienne \& Jacquet, 1983; Metcalf \& Chalk, 1972; Mainieri \& Chimelo, 1989), exceto para os vasos de maior diâmetro, o parênquima axial difuso e os raios largos e altos, próprios das lianas (Carlquist, 1991). Pontoaçôes intervasculares guarnecidas e fibras septadas, comuns na família não foram observadas. A semelhança do lenho das duas espécies é perceptível, sendo os vasos difusos, com diâmetro uniforme e largos (>309 $\mu \mathrm{m})$, arredondados, predominância de solitários, pouco numerosos $\left(6-10 / \mathrm{mm}^{2}\right)$, curtos a muito longos $(301-1000 \mu \mathrm{m})$ e com pontoaçôes arredondadas. O parênquima axial é apotraqueal difuso em agregados, unilateral distinto ou quase distinto sob lente e o radial unisseriado, coincidente com Silva et al., (1998), e com Zevallos et al., (2002) somente em U. guianensis (indicou multisseriados para $U$. tomentosa), heterocelulares, número de células (7-42), largos (101-200 $\mu \mathrm{m})$, medianos a moderadamente altos $(2-20 \mathrm{~mm})$, poucos a muito numerosos (3 a $>29 / \mathrm{mm})$. As fibras mostram pontoaçōes areoladas, maiores de $3 \mu \mathrm{m}$, médias a medianamente longas $(0,9-2,2 / \mathrm{mm})$, estreitas a largas $(<24 \mathrm{a}>40 \mu \mathrm{m})$ e com paredes muito delgada a espessa. As diferenças das espécies, encontram-se na largura dos raios, na proporção de vasos solitários e na freqüência dos mesmos; podendo ser resultado do hábito de crescimento e habitat das plantas: $U$. guianensis, de hábito lianescente com tendência a arbustiva, em florestas secundárias e $U$. tomentosa, de hábito lianescente, em florestas primárias (Zevallos et al. 2000), relação que destaca nas lianas (Carlquist, 1985).

A descrição da estrutura anatômica do lenho das duas espécies de lianas, segundo Gentry (1993), tem importante aplicação na sua identificação taxonômica, constituindo valiosa informação complementar aos elementos reprodutivos, geralmente empregados nos trabalhos de campo e nos herbários.

\section{CONCLUSÕES}

A estrutura anatômica macro e microscópica do lenho das plantas de Uncaria tomentosa e de U. guianensis mostraram características peculiares à Família Rubiaceae, à exceção dos elementos vasculares solitários, arredondados, largos e freqüentes, parênquima axial difuso e largura dos raios, que correspondem a plantas de hábito lianescente. Os parâmetros anatômicos do lenho das duas espécies, de forma complementar à morfologia dos órgãos vegetativos e reprodutivos, permitem a identificação das plantas em condições de campo e de laboratório.

\section{AGRADECIMENTOS}

A Maria A. R. Bermúdez pelo preparo das lâminas histológicas do lenho; aos Profs. Evandro Ferreira, Marcos Silveira e Silvia Brilhante da Universidade Federal do Acre pelo acesso ao Laboratório do HPZ; ao Pesq. Geraldo J. Zenid, do IPT/São Paulo pelo apoio nas descrições anatômicas do lenho; a OEA pela bolsa-de-estudos para o desenvolvimento da Tese de Doutorado, do $1^{\circ}$ autor do artigo.

\section{BIBLIOGRAFIA CITADA}

Aquino, R.; Simone, F.; Pizza, P.; Conti, C.; Stein, L.M. 1989. Plant metabolites: structure and in vitro antiviral activity of quinovic acid glycosides from Uncaria tomentosa and Guettarda platypoda. Journal of Natural Products, 52:679-685.

Berlyn, G.P. 1976. Botanical microtechnique and cytochemistry. The Iowa University. Iowa. 454pp.

Cabieses, F. 1997. La uña de gato y su entorno. 2nd ed. Universidad Particular San Martín de Porres. Lima, Perú. 145pp.

Carlquist, S. 1985. Observations on funcional wood histology of vines and lianas: vessel dimorphism, tracheids, narrow vessels, and parenchyma. Aliso 11:139-157.

Carlquist, S. 1991. Anatomy of vine and liana stems: review and synthesis. In: The biology of vines (eds. Putz, F.E.; Mooney, H.A..): 53-71. Cambridge University Press, Cambridge.

Chavesta, C.M.; González, M.E. 1996. Breve descripción anatómica de la madera de Uncaria guianensis y Uncaria tomentosa. Universidad Nacional Agraria La Molina. Lima, Perú. 6p.

Comissão Panamericana de Normas Técnicas-COPANT. 1974. Descrição macroscópica, microscópica e geral da madeira. São Paulo. COPANT, v. 30, p.1-19.

Coradin, V.T.R.; Muniz, G.I de. 1992. Normas e procedimentos em estudos de anatomia da madeira: Angiospermae II-Gimnospermae. Série Técnica 15. IBAMA. DIRPED.LPF. Brasília. 17pp.

Desmarchelier, C.; Mongelli, E.; Coussio, J.; Ciccia, G. 1997. Evaluation of the antioxidant activity in extracts of Uncaria tomentosa. Phytother Research, 11:254-256.

Détienne, P.; Jacquet, P. 1983. Atlas d'identification des bois de l'amazonie et des régions voisnes. Centre Technique Forestier Tropical. Montpellier, France. 279pp.

Franklin, G.L. 1937. Permanent maceration of macerated wood fibers. Tropical Woods, 9:21-22.

Freitas, A.J.; Vasconcellos, F.J.; Silva, B.N.; Loureiro, A.A. 1992. Madeiras da Amazônia que apresentam raios largos. Acta Amazonica, 22(1):91-161.

Gentry, A. 1993. A field guide to the families and genera of woody plants of northwest Southamerica. Conservation Internacional. Washington DC. 895pp.

Huang, K.C. 1999. The pharmacology of Chinese herbs. Hardcover Book Press Co. Florida. 125pp.

IAWA Committee. 1989. IAWA list of microscopic features for hardwood identification. IAWA Bulletin, 10(3):219-332.

Instituto Peruano de Seguridad Social-IPSS. 1997. Plantas medicinales de la Amazonía Peruana. INMETRA. Iquitos-Peru. 285pp.

Jansen, S.; Robbrecht; H.E.; Beckman, H.; Smets, E. 2002. A survey of the systematic wood anatomy of the Rubiaceae. IAWA Journal, 23(1):1-68.

Johansen, D. 1940. Plant microtechnique. MacGraw-Hill Book, New York, 450pp.

Keplinger, K.; Laus, G.; Wurm, M.; Dierich, M.P.; Teppner, H. 1999. Uncaria tomentosa (Willd.) DC.- ethnomedicinal use and new pharmacological, toxicological and botanical results. Journal of Ethnopharmacology, 64:23-34. 
Lindorf, H. 2001. Reconocimiento al microscopia de la corteza y el polvo farmaceutico de la "uña de gato": $1^{a}$ Reunión Internacional del Género Uncaria "uña de gato". Pontificia Universidad Católica del Perú. Iquitos, Perú. 23pp.

Lock de Ugaz, O. 1994. Investigación fitoquímica: Uncaria tomentosa y U. guianensis. Lima, Pe: Fondo Editorial, Pontificia Universidad Católica del Perú. 300p.

Mainieri, C.; Chimelo, J.P. 1989. Fichas de características das madeiras brasileiras. 2. ed. São Paulo: IPT, 418p. (Publicação, 1791).

Metcalfe, C.R.; Chalk, L. 1972. Anatomy of the Dicotyledons. Oxford Claredon Press. Oxford, 1500pp.

Obregón, L.E. 1995. "Uña de gato”, género Uncaria: estudios botánicos, químicos e farmacológicos de Uncaria tomentosa e Uncaria guianensis. Instituto de Fitoterapia Americano. Lima, Perú. 64pp.

Radford, A. E.; Willians, C.; Dickson, J.; Massey, R.; Ritchie, B.C. 1974. Vascular plant systematics. Harper and Row. New York, 891pp.

Record, S.J.; Hess, R.W. 1949. Timbers of the New World. Yale University Press. New Haven, 640pp.

Reinhard, K.H. 1999. Uncaria tomentosa (Willd.) D.C.: cat's claw, una de gato, or saventaro. Journal Alternative Complement Medicine, 5:143-151.

Ridsdale, C.E 1978. A revision of Mitragyna and Uncaria. Blumea, 24:43-100.

Sass, J.E. 1951. Botanical microtechnique. 2ed. State College Press. Iowa. 350pp.

Silva, D.H.; Alvarado, D.R.; Hidalgo, H.J.; Cerrutti, S.T.; Garcia, R.J.; Dávila, M.W.; Mestanza, D.M.; Rios, I.F.; Nina, C.E.; Nonato, L.R.. 1998. Monografia de Uncaria tomentosa (Willd.) DC. Instituto Peruano de Seguridad Social-Instituto de Medicina Tradicional. Iquitos, Perú. 103p.

Steyermark, J.A. 1974. Rubiaceae. In: Flora de Venezuela. (ed. Laser, T.) Instituto Botánico, Dirección de Recursos Naturales Renovables, Ministerio de Agricultura y Cria, Caracas, 9: 12070 .
Tratado de Cooperación Amazónica-TCA. 1995. Plantas medicinales Amazónicas: realidades y perspectivas. Secretaria Pro-Tempore. Lima-Perú. 148pp.

Urrunaga, S.R. 1994. Uncaria tomentosa (uña de gato): un recurso fitogenético valioso del Perú. Centro de Plantas MedicinalesUniversidad Nacional de San Antonio Abad del Cusco. Cusco, Perú. 66pp.

Villachica, H.; Lazarte, J.; Clavo, M.; Lescano, C.; Arroyo, M.; Diaz, I. 1998. Productos amazónicos del Perú: palmito, camucamu y uña de gato. Consorcio Para el Desarrollo Sostenible de Ucayali-CODESU. Pucallpa, Peru. 115pp.

Wang, Z. 1989. Quantitative determination of the alkaloids of $U$. rhynchophylla by calcium chromatography. ChineseTraditional and Herbal Drugs, 20:11-20.

Zavala, A.; Zevallos, P.P. 1996. Taxonomía, distribuición geográfica y status del gênero Uncaria en el Perú. Faculdad de Ciencias Forestales, Universidade Nacional Agraria La Molina. Lima, Perú. 78pp.

Zevallos, P.P.; Lombardi, I.; Bernal, Y. 2000. Agrotecnología para el cultivo de la uña de gato o bejuco de agua: Fundamentos de agrotecnología para el cultivo de plantas medicinais Iberoamericanas. Convenio Andrés Bello/Ciencia y Tecnología para el Desarrollo. Santafé de Bogotá, Colombia. 90pp.

Zevallos, P.P.; Chavesta, C.M.; Buitron, D.G.; Takashiba, H.E. 2002. Determinação de contenidos oxindolícos de espécies del género Uncaria Schreb. "uña de gato" a través de la correlación de características foliares y anatómicas de la madera en tres localidades de la amazonia peruana Resumenes del $8^{\circ}$ Congreso Latinoaméricano de Botánica. ALB/ACB/ACH/ICN. Santafé de Bogotá, Colombia.

Recebido em 03/07/2004

Aceito em 23/03/2006 